\title{
Multi-wavelength transmittance photoplethysmography with near infrared laser diodes during exercise
}

\author{
S. M. López Silva ${ }^{\mathrm{a}}$, R. Giannetti ${ }^{\mathrm{b}}$, M. L. Dotor ${ }^{\mathrm{c}}$, D. Golmayo $^{\mathrm{d}}$, P. Martín ${ }^{\mathrm{e}}$, F. Miguel-Tobal $^{\mathrm{e}}$, A. \\ Bilbao $^{\mathrm{e}}$, J. P. Silveira ${ }^{\mathrm{c}}$ \\ ${ }^{a}$ Instituto Universitario de Microelectrónica Aplicada, Universidad de Las Palmas de Gran Canaria, \\ Spain \\ ${ }^{\mathrm{b}}$ Departamento de Electrónica y Automática, Universidad Pontificia Comillas de Madrid, Spain \\ ${ }^{\mathrm{c} I n s t i t u t o}$ de Microelectrónica de Madrid, CNM, CSIC, Spain \\ ${ }^{\mathrm{d}}$ Instituto de Ciencia de Materiales de Madrid, CSIC, Spain \\ ${ }^{\text {e}}$ Escuela Profesional de Medicina de la Educación Física y el Deporte, Universidad Complutense de \\ Madrid, Spain
}

\begin{abstract}
The transmittance photoplethysmographic signals recorded with multiple NIR laser diodes in athletes along a maximal exercise test by treadmill ergometer and the results after processing are presented in comparison to the established reference techniques.
\end{abstract}

Keywords: Diode lasers; Medical optics instrumentation; Optical diagnostics for medicine

\section{INTRODUCTION}

Photoplethysmography ${ }^{1,2}$ is a technique based on optical principles, which monitors the heart rate. Pulse oximetry ${ }^{3-5}$ combines the photoplethysmography with the hemoglobin optical absorption characteristics ${ }^{6,7}$ through the analysis of luminous signals of specific wavelengths. Thus, this non-invasive technique is widely used to determine the peripheral oxygenation degree and the heart rate. The classical devices use two light emitting diodes as sources, with emissions in the red $(630-660 \mathrm{~nm})$ and in the infrared $(880-940 \mathrm{~nm})$ regions of the optical spectrum.

There it is a great interest to monitor the heart rate and the oxygen saturation during exercise in order to improve the sport training $3,8,9$. The main problem is the apparition of movement artefacts ${ }^{3,9}$. Here we present the results obtained after the analysis of photoplethysmographic signals recorded in athletes along maximal exercise tests by treadmill ergometer, in comparison to the standard techniques. We used a new transmittance sensor based on three laser diodes (LD) emitting at near-infrared wavelengths (NIR), in a measurement system with a processing algorithm previously reported $^{10}$.

\section{MATERIALS AND METHODS}

\subsection{Measurement system}

Our measurement system (figure 1) is composed by a new optical sensor, the sensor electronics, and a data acquisition (DAQ) board and programs installed in a portable personal computer (PC). The vascular bed to be analyzed is fixed between the emitters and the detection side of the sensor in a transmittance configuration. The new optical sensor was developed with three laser diodes emitting close to $850 \mathrm{~nm}$ (LD1), $780 \mathrm{~nm}$ (LD2), and $980 \mathrm{~nm}$ (LD3). The lasers are mounted on a single metal substrate and nearer, in order to illuminate a similar finger area with each one of them. This fact minimizes the influence of motion artifacts on the measurement. The sensor detection side consists of three silicon p-i-n de BPW34 photodiodes connected in parallel to increase the detection area and the first amplification stage in their backside. The laser-diodes driver, amplification stages, timing and sample and hold $(\mathrm{S} \& \mathrm{H})$ circuits constitute the sensor electronics. Each LD is activated with pulses of $10 \mu \mathrm{s}$ in duration at $1 \mathrm{kHz}$ of repetition rate. The outputs of the S\&H are fed into the analogue inputs of a 12-bit DAQ board. This board and the processing programs are installed on the PC. The S\&H output signals are analogically pre-filtered before the DAQ with a simple anti-aliasing RC low pass filter at 
$300 \mathrm{~Hz}$ and then digitized at $1 \mathrm{kSa} / \mathrm{s}$ for every channel. The next stages of the signal processing are carried out digitally, either in real time or offline (post-processing), after a ten-sample averaging of each wavelength PPG signal. An algorithm ${ }^{10}$ that comprises a non-linear filtering with a frequency domain analysis has been developed in order to obtain a valid value of the heart rate from the recorded PPG.

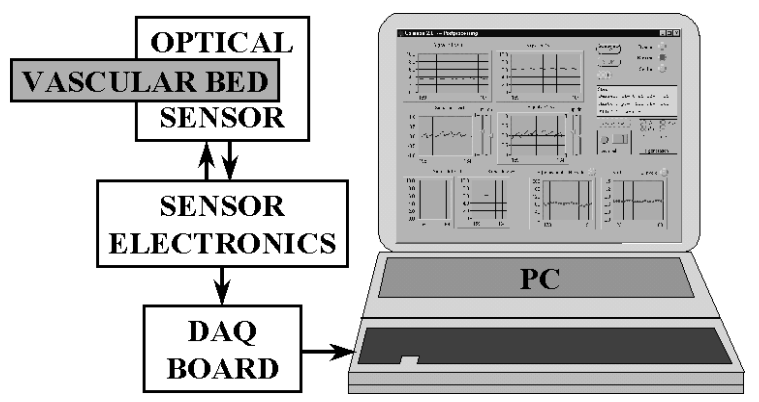

Figure 1: The measurement system is composed by the near infrared laser diodes based optical sensor, which is fixed to a fingertip (vascular bed), the sensor electronics and the personal computer with the data acquisition board and the programs.

To obtain a valid measurement, a method that composes the peculiar non-linear filtering used for the oximeter ${ }^{12}$ with an on-line frequency domain analysis has been designed. A simple filtering is not viable in this case, given the fact that the athletes tend to run or move with a rhythm that is near a multiple of their heartbeat when they reach maximum exercise conditions, so that filtering out the movements artifact is not trivial at all. Movement artifacts affect to oximeter much more than to ECG system, given the fact that the latter instrument uses strongly fixed electrodes which can be assumed to not move during the exercise, and that the quantity measured is not affected in noticeable way by the relative positions of the electrodes with the body. On the contrary, oximeter measurement is based on the light transmittance coefficient of a tissue with arterial blood circulation, and although mid-term relative position change will not have any influence, short-term (less than one heartbeat) ones will affect the measurement. In literature, it is suggested the utilization of reflection-type oximeter ${ }^{11}$ which could be placed in zones which moves a lot less, as the head or trunk.

The proposed method allows us to obtain the heartbeat value during the exercise, even during the treadmill test. This method is based on the observation that, while the normal photoplethysmographic signals have a quite low harmonic content, the signal produced by the movement could be characterized by a high harmonic content. In fact, this signal is produced basically by the variation of the distance between lasers and receptors in the finger, which in turn is mainly an effect of the impulsive movement of the athlete's strides, and the jumping involved.

The processing algorithm is summarized as follows. From the data flow representing the photoplethysmographic signal (already filtered through anti-aliasing, Bessel band-pass and a median-like filter ${ }^{12}$ ) a sliding window of a ten-second worth of data is selected. A Fast Fourier Transform (FFT) is applied to this data in order to obtain the power spectra of the signal. The next step is to apply a peak-search algorithm, designed to be robust and to merge near-peak probably due to noise, which outputs a list of frequency-value pairs representing possible candidates for the hearth-beat signals. In the following phase, the algorithm compares pairs of peaks (and triplets of them if necessary), trying to identify — via a heuristic function comparing the value of the peak's frequencies - components of the same periodic signal. The last step takes into account the signals found, their harmonic contents and the previous value of heart beat and select the most-probable heart beat value. This computation is performed in a near-background and parallel fashion, via a careful interleaving with the data acquisition and the linear filtering and processing. On the PC where it is implemented (a portable, Pentium-I based architecture) we are able to obtain one data point every two seconds.

The "front panel" of the resulting virtual instrument working in post-processing mode is shown in fig. 2. It is possible to set-up processing parameters (right upper part of fig. 2) such as Bessel filters cut-off frequencies (f1 and f2), histogram type non-linear filter window and cut-off points (pt and vl). Here are depicted the whole raw PPG signals and their variable components after filtering of up to four channels or emitters (four boxes in the left upper part of fig. 2), and their frequencies as heart beats per minute (BPM, four boxes in the left middle part of fig. 2). In the lower part of figure 2, from left to right, are shown the main FFT channel and its frequency values as heart beats per minute in the box to the 
left (FFT HB), the record of heart beats (HB) over the last 49 seconds and its validity, as well as some quotients (Q vs. t) obtained from the constant and variable components of two PPG channels, related to the oxygen saturation.

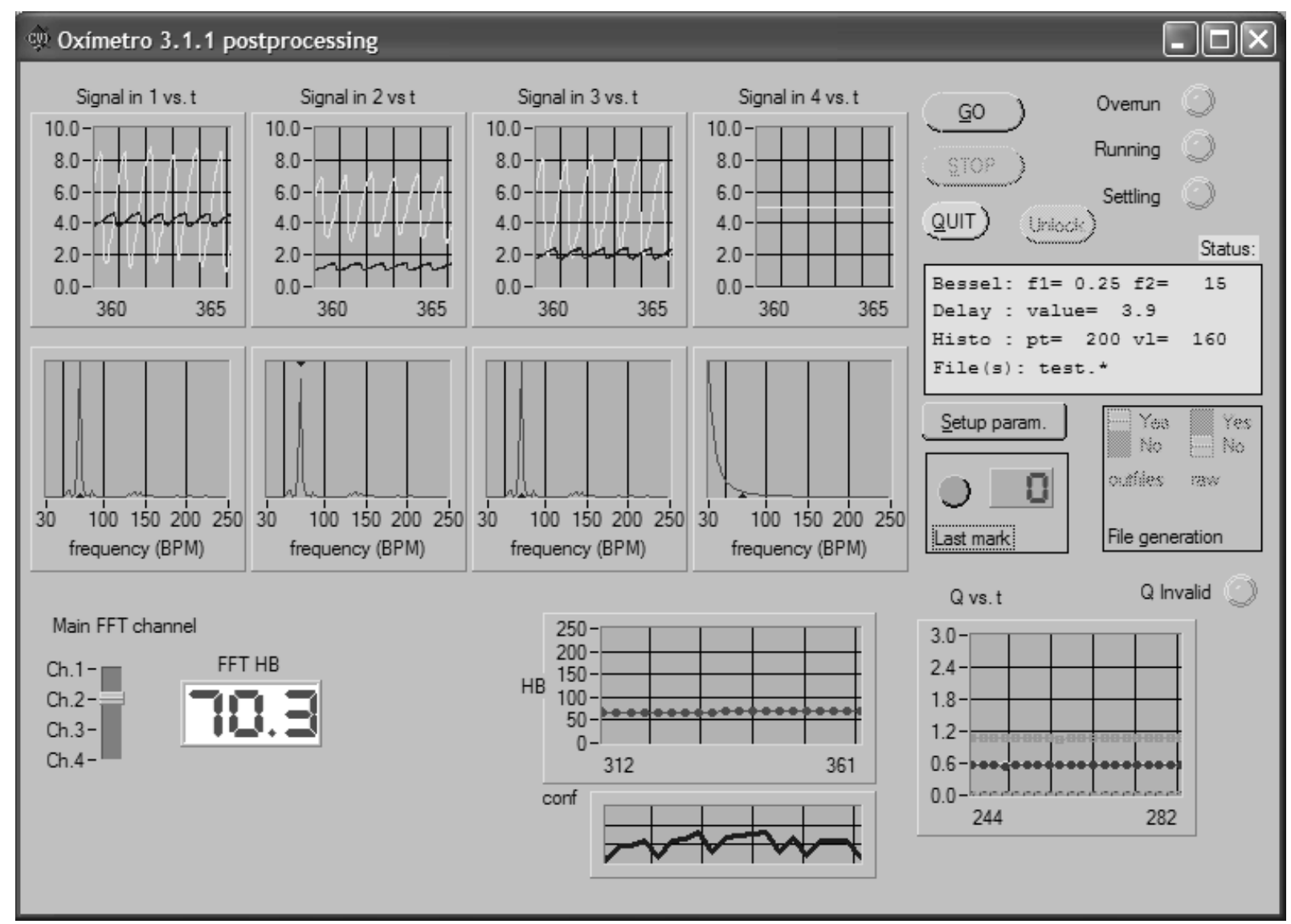

Figure 2: Front panel of the resulting virtual instrument. See text for explanation.

\subsection{Subjects and experimental protocol}

In order to test the stability of our new three laser diodes sensory system and the performance under slight breath variations a preliminary test was conducted on a healthy 42 -years female volunteer. The sensor was fixed to a right hand finger and the volunteer sited keeping this right hand resting on. Raw transmittance photoplethysmographic signals were recorded over basal conditions and along breathing maneuvers.

A prospective study was conducted on several white or Caucasian female and male athletes, with an age included between 18 and 44 years, and whose training schedule exceeded an average 7-8 hours per week. Prior to commencing the study, the athletes were informed about the test and gave written consent. Tests were performed used a treadmill ergometer. The athletes were connected to measurement instruments before starting the exercise, and then a standard exercise was programmed on the machines. Measurements included electrocardiography, blood pressure, oxygen saturation (with a standard commercial pulse oximeter), heart rate by photoplethysmography (with a standard commercial pulse oximeter and with our NIR-laser prototype one), and exhaled gases real-time analysis with a breathby-breath analyzer SensorMedics Vmax29c. Two different prototypes of our NIR-laser sensor were used alternatively, one with two lasers (LD1 - $850 \mathrm{~nm}$ and LD3 - $980 \mathrm{~nm}$ ) and a second three-lasers prototype (LD1 - $850 \mathrm{~nm}$, LD2 - 780 $\mathrm{nm}$, and LD3 - $980 \mathrm{~nm}$ ). The fixation of the sensors was made — for both the experimental instrument and the commercial one - to a finger of the athlete. 
The measurements were performed at the laboratories of Professional School of Sport Medicine at the Complutense University of Madrid. Each of the subjects was prepared by connecting the measurement electrodes and tubes, and then a complete maximal exercise test was performed. Pressure data was obtained before and after the exercise. A complex protocol was used. The athlete started (after warming up) at $6 \mathrm{~km} / \mathrm{h}$ and $1 \%$ slope, and the speed was increased every 2 minutes by $2 \mathrm{~km} / \mathrm{h}$ until $16 \mathrm{~km} / \mathrm{h}$ were reached; afterwards speed was maintained constant, slope was increased to $3 \%$, and maintained until the maximum effort conditions were attained.

\section{RESULTS AND DISCUSSION}

In figure 4 are shown the heart rates (beats per minute, bpm) obtained from two male athletes (a, APm05 and b, AOm05) over the whole maximal exercise test by treadmill ergometer, using electrocardiography (ECG), a commercial pulse oximeter (CPO) and our system with two laser diodes (LD1 and LD3). In the first case (a, APm05) both of our NIR LDs follow very well the ECG behavior pattern until the maximal effort around $900 \mathrm{~s}$, and with some disparities after this moment until the end (recovery phase). By contrast, the CPO only follows the ECG in the initial part of the test (below $200 \mathrm{~s}$ ), as shown in figure 4a. In the second case (b, AOm05) the four sets of experimental points almost agree, although some points (of LD1, LD3 and CPO) are out of the ECG pattern.

Figure 5 represents the raw transmittance photoplethysmographic signals (a, b, c, d) recorded over 300 seconds (a, b) and 10 seconds $(\mathrm{c}, \mathrm{d})$ time intervals and the heart rates (e and $\mathrm{f}$, beats per minute, bpm) over the $300 \mathrm{~s}$ interval, obtained from a female volunteer over basal conditions (a, c, e, MLf53) and through breathing maneuvers (b, d, f, MLf52) with our laser diodes based system (LD1, LD2, and LD3). The recorded PPG signals over basal conditions are stable with some oscillations probably associated to respiration (fig. 5a) and have a large variable component (fig. 5c). The mean values and standard deviation (Mean and SD, respectively in fig. 5e) of the 117 (Size) heart rate values considered in the $300 \mathrm{~s}$ time interval are similar for the three laser diodes and have a very low variation. To perform the breath maneuvers the volunteer took a deep breath ( $\mathrm{db}$ in fig. $5 \mathrm{~b}$ ) prior to hold one's breathe some seconds. Then, 90 seconds later, the volunteer held breath again. The recorded PPG signals over the second breath maneuver have a low variable component (fig. 5d), are more irregular than those for basal conditions, and the maneuvers are reflected in their signal values (fig. $5 b)$. Finally, the breath maneuvers caused changes in the heart rate values, as shown in figure $5 f$.

In figure 6 are depicted the heart rates (beats per minute, bpm) obtained from a female (a, SZf04) and a male (b, EQm05) athletes over the whole stress test by electrocardiography (ECG), a commercial pulse oximeter (CPO) and our laser diodes based system (LD1, LD2, and LD3). In both cases (fig. 6, a, SZf04 and b, EQm05) the heart rate values obtained with our NIR LD system are similar to those from the ECG over the whole experiment, while the CPO coincides with ECG only at the beginning (below $200 \mathrm{~s}$ ) of the female athlete experiment (fig. 6a). During the rest of the female athlete experiment and over the whole male athlete experiment (fig 6b), the CPO underestimates and sometimes overestimates the heart rate in comparison with the ECG record.

It is possible to appreciate the variations of the raw transmittance PPG signals recorded in different moments of the tests in figure 7. The photoplethysmogramms in the 10 -second time interval from $50 \mathrm{~s}$ to $60 \mathrm{~s}$ in figure $7 \mathrm{c}$ corresponds to the first seconds of the male athlete test, being the signals very uniform and periodical with a high pulsatile component. The signals from $355 \mathrm{~s}$ to $365 \mathrm{~s}$ of figure $7 \mathrm{a}$ correspond to a more advanced phase of the female athlete test, and show lower and higher variations associated to the cardiac pumping action and with the athlete movement. The PPG of figure $7 \mathrm{~b}$ and $7 \mathrm{~d}$ correspond to the phase of maximal effort in both tests of the female (SZf04) and male (EQm05) athletes, in which are present stronger movements, and the variations of the signals are less uniform.

During the preparation and warm-up phase, the measurement presents no problem, and the three main instruments the ECG meter, the commercial pulse oximeter and our NIR-laser prototype — give the same measurement (in the acceptable error range) for the hearth rate. On the other hand, when the athlete is working at full power and in a more noticeable way when he (or she) is reaching exertion, the measurement by the two photoplethysmographic devices is difficult to obtain. In fact, the frantic movement of big muscular groups causes a false signal on the measured quantity. This is due to the relative movement between sensors and the athlete's fingers, and by physiological deformation of the (elastic) tissues caused by the movement itself ${ }^{11}$. Generally, this effect is much more noticeable in the treadmill test, because in this case we not only have the fast movement of the legs, but we also have to take into account the bouncing of the body on the carpet and the movement of the arms which may create local blood accumulations in the hands. Also, when the athlete is coming to maximal point, he (or she) jumps to go out the treadmill and then hold onto the lateral security handrails. This last might cause additional disturbances in the recorded PPG signals, and indeed, wrong heart rate values in the hand-finger photoplethysmographic devices, in both the CPO and our NIR-LD prototype. 


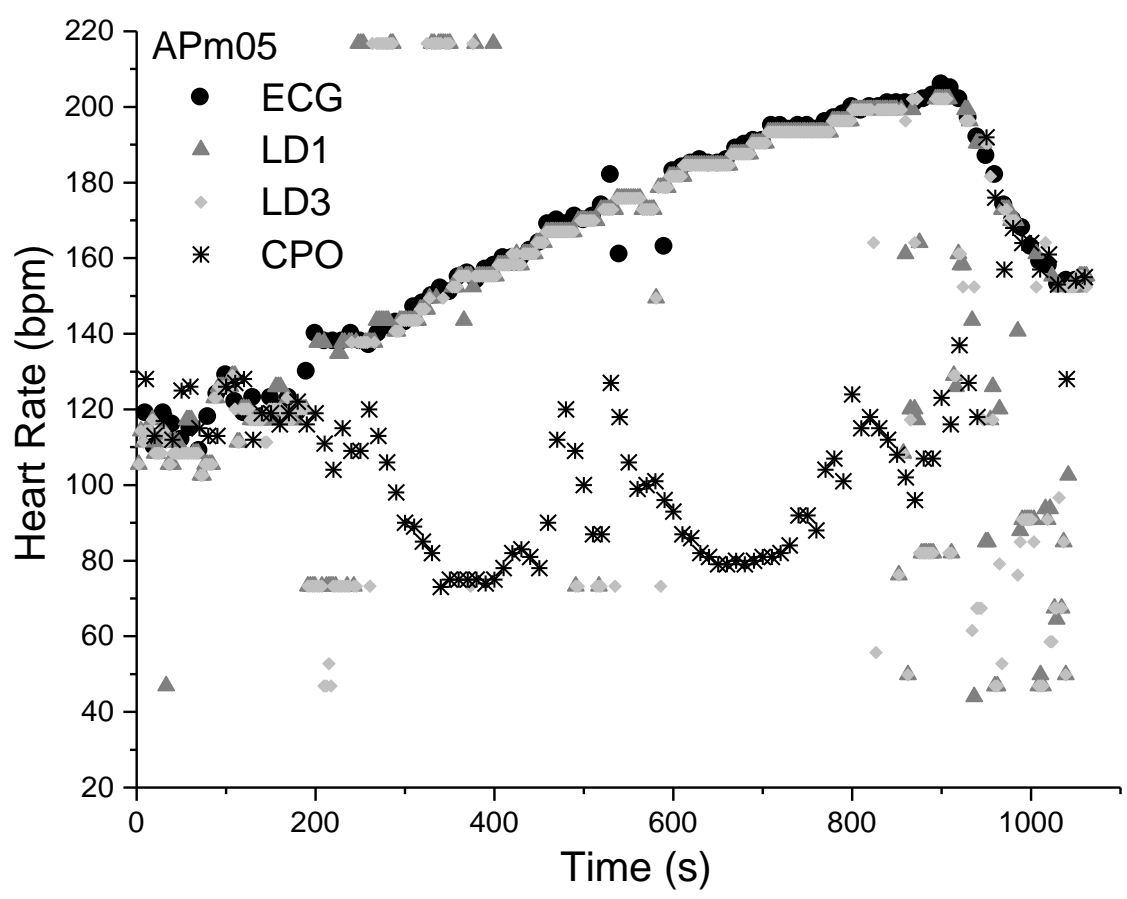

(b)

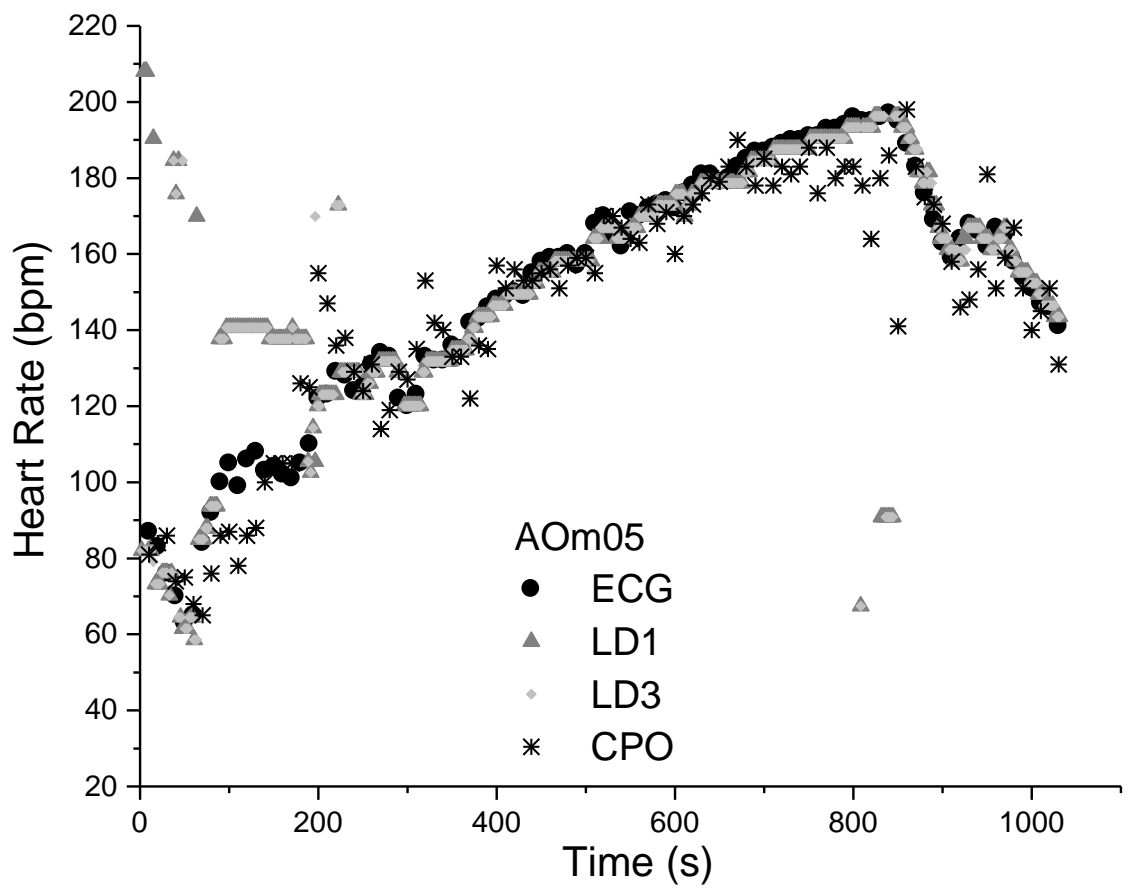

Figure 4: The heart rates (beats per minute, bpm) obtained from two male athletes (a, APm05 and b, AOm05) over a stress test with electrocardiography (ECG), a commercial pulse oximeter (CPO) and our system with two laser diodes (LD1 and LD3). 
(a)

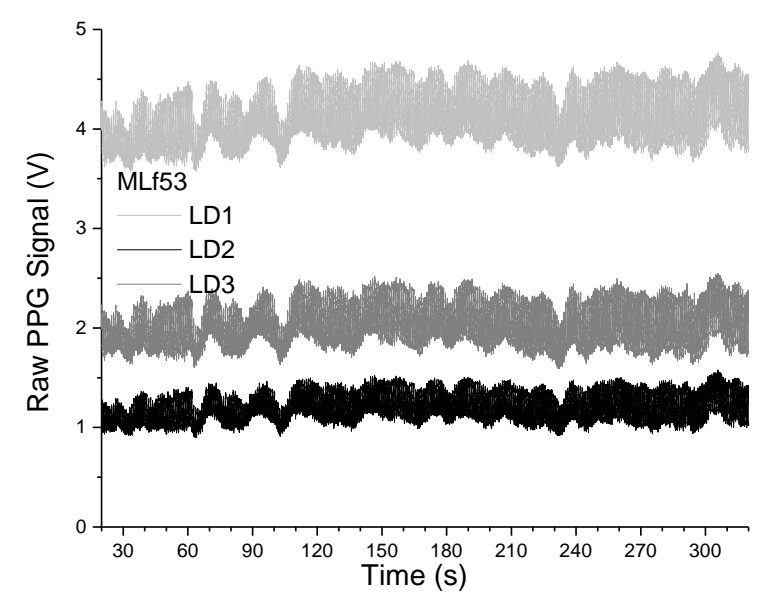

(c)

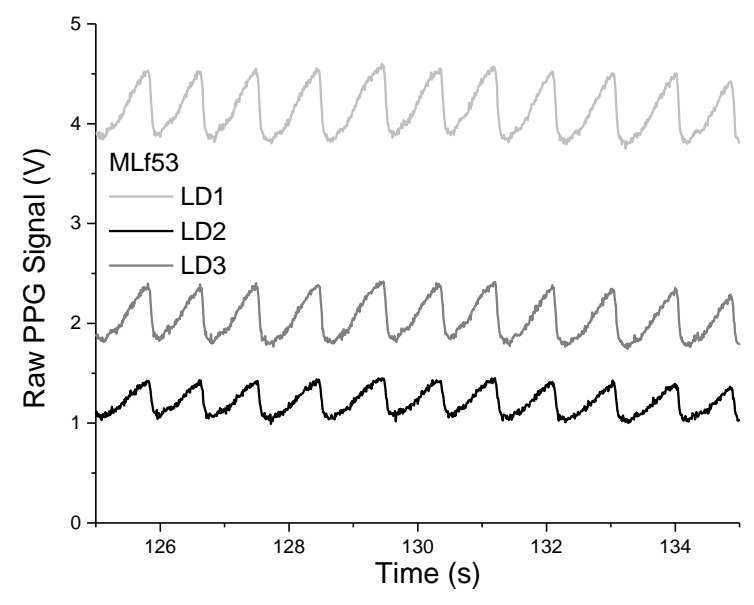

(e)

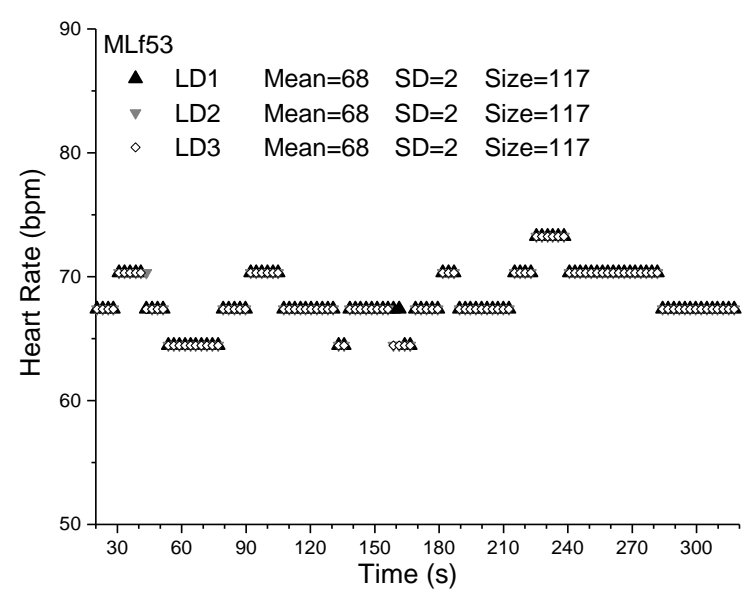

(b)

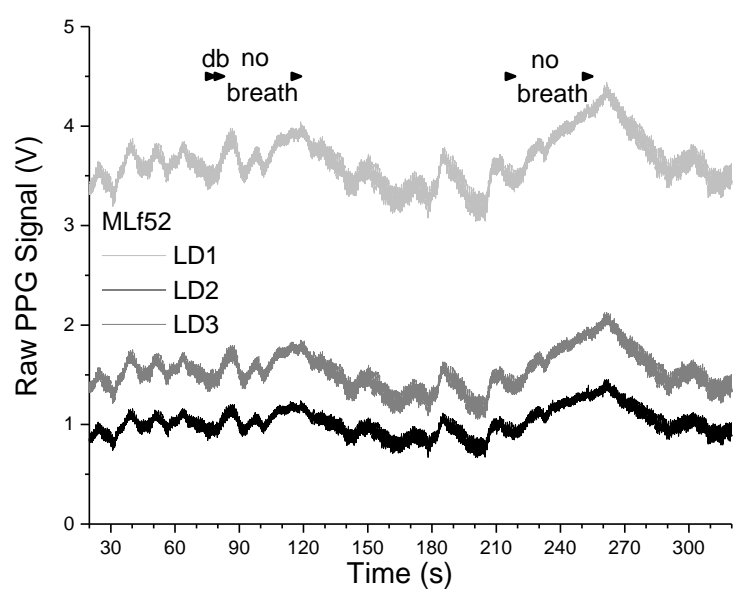

(d)

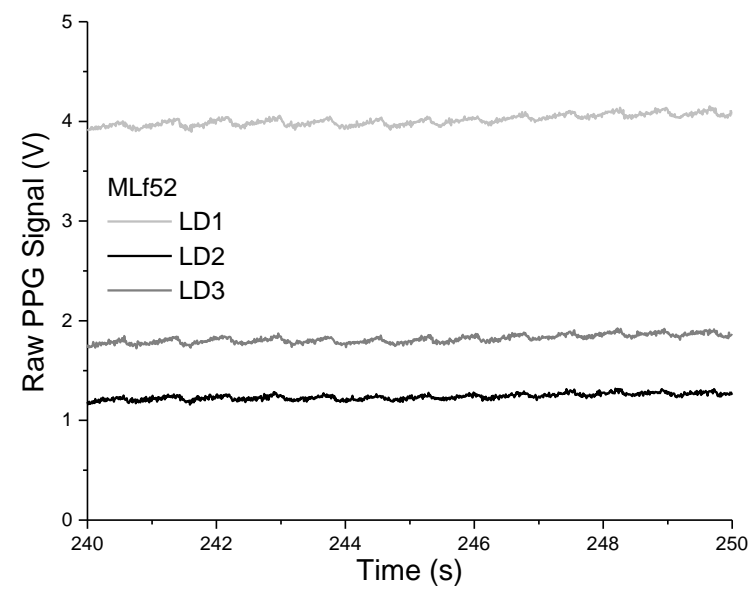

(f)

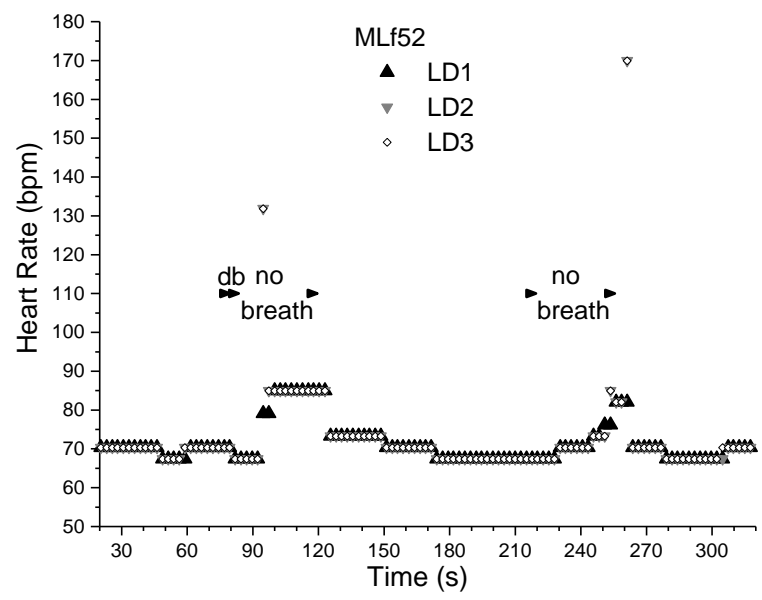

Figure 5: Raw transmittance photoplethysmographic signals (a, b, c, d) recorded over $300 \mathrm{~s}(\mathrm{a}, \mathrm{b})$ and $10 \mathrm{~s}(\mathrm{c}, \mathrm{d})$ time intervals and the $300 \mathrm{~s}$ heart rates (e and f, beats per minute, bpm) obtained from a female volunteer over basal conditions (a, c, e, MLf53) and through breathing maneuvers (b, d, f, MLf52) with our laser diodes based system (LD1, LD2, and LD3). 
(a)

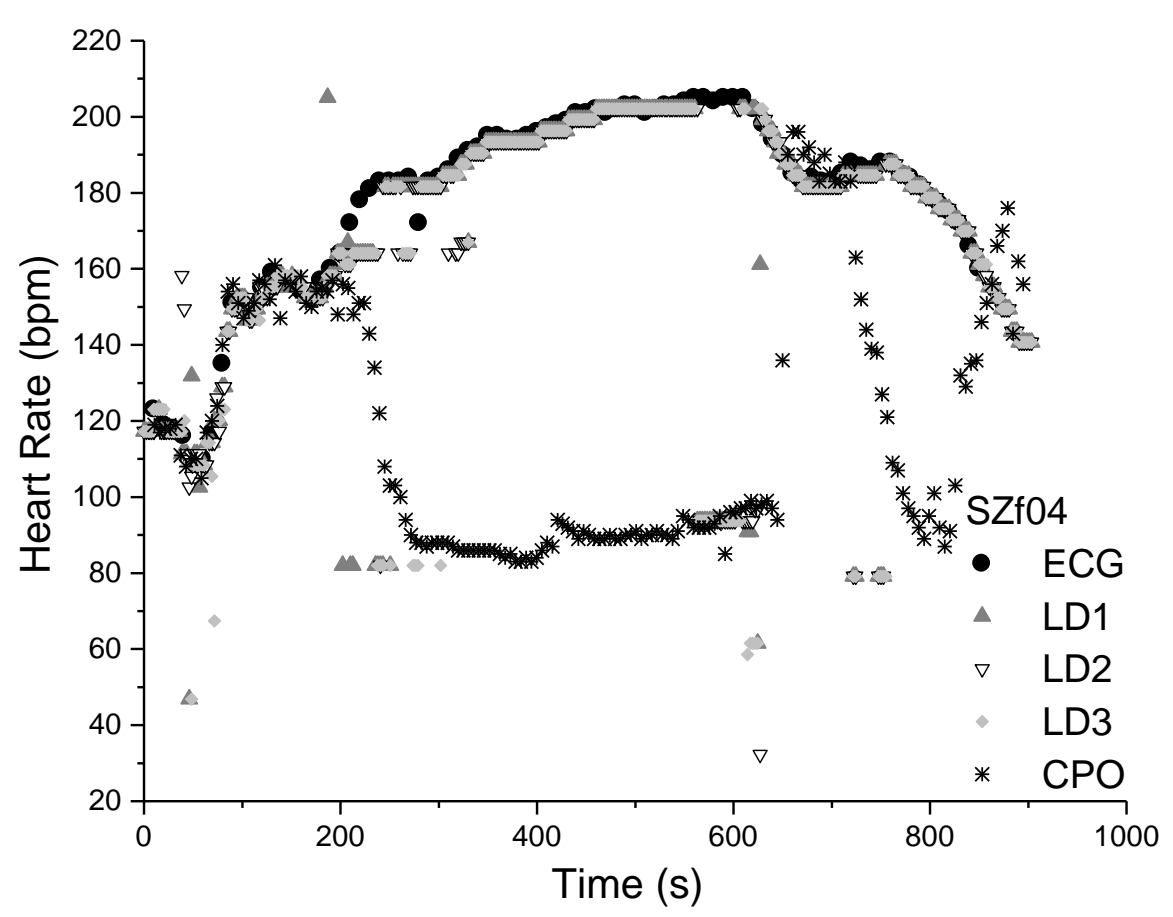

(b)

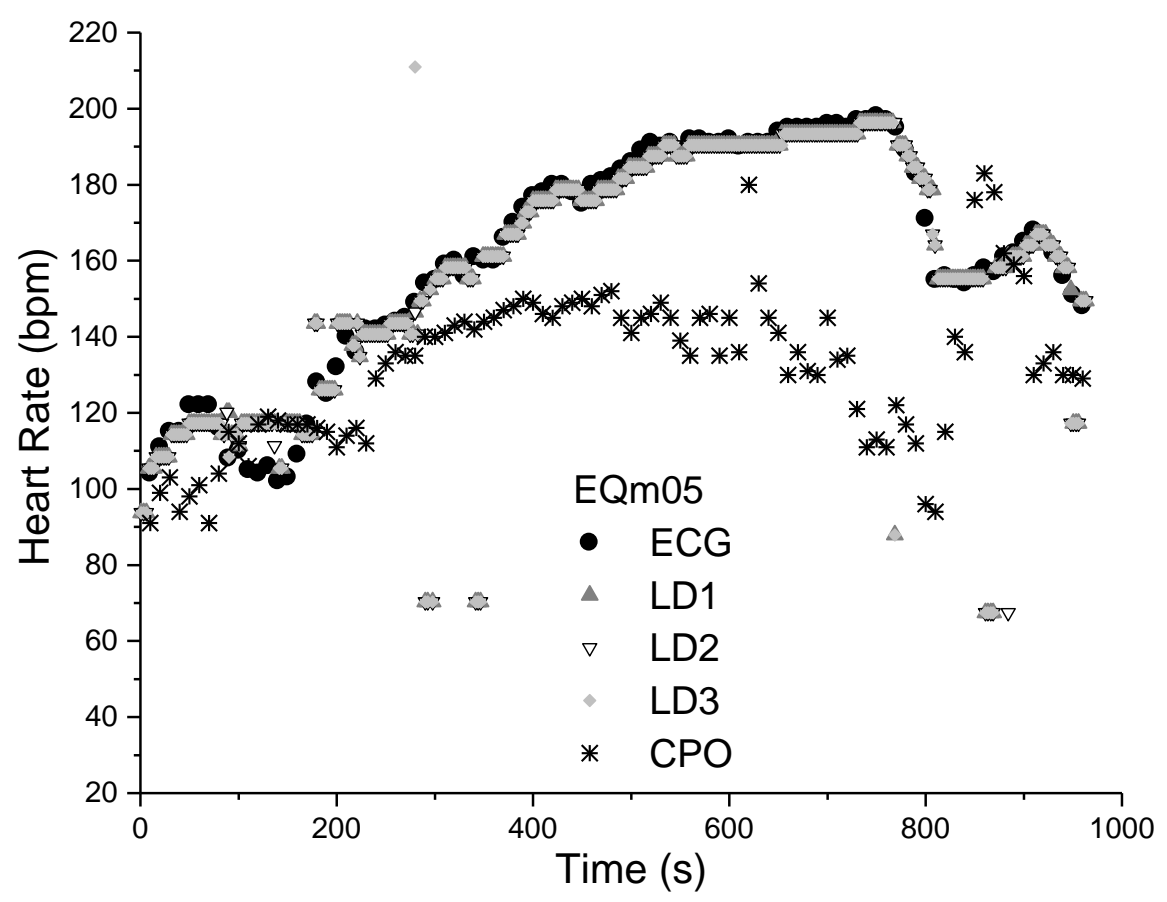

Figure 6: The heart rates (beats per minute, bpm) obtained from a female (a, SZf04) and a male (b, EQm05) athletes over a stress test with electrocardiography (ECG), a commercial pulse oximeter (CPO) and our laser diodes based system (LD1, LD2, and LD3). 
(a)

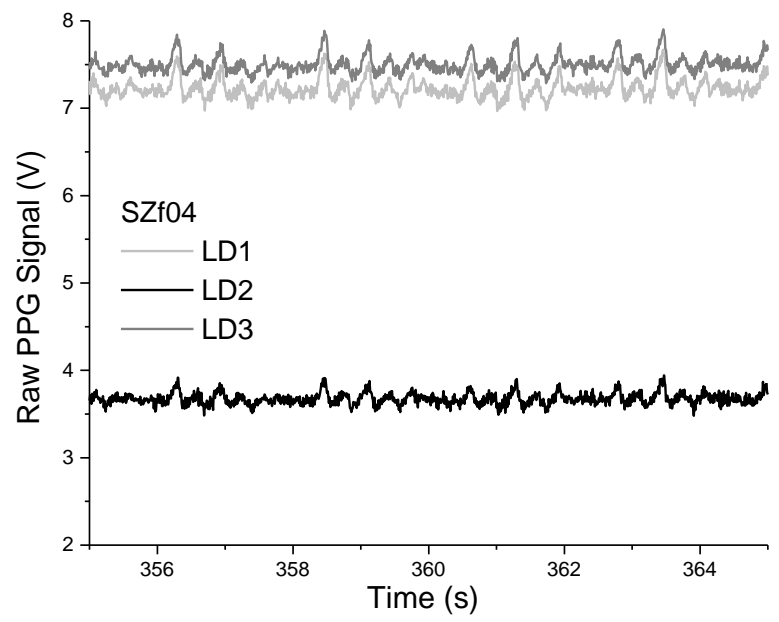

(c)

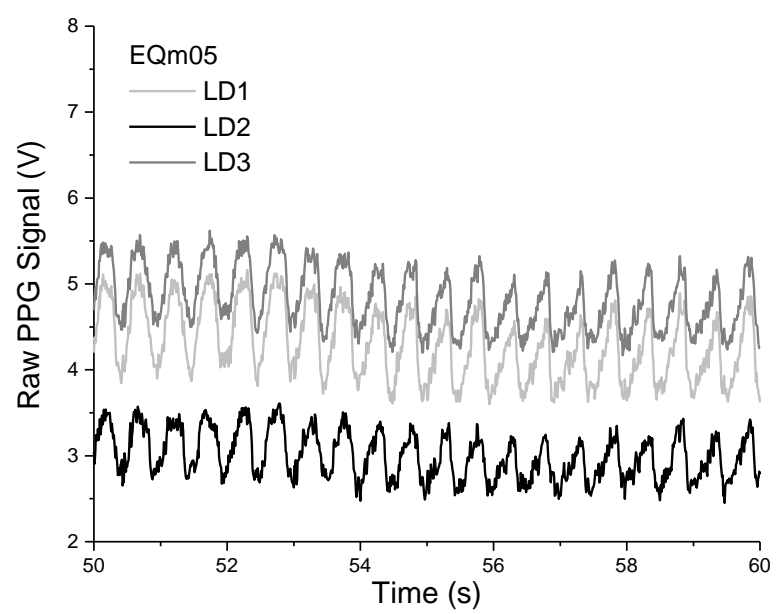

(b)

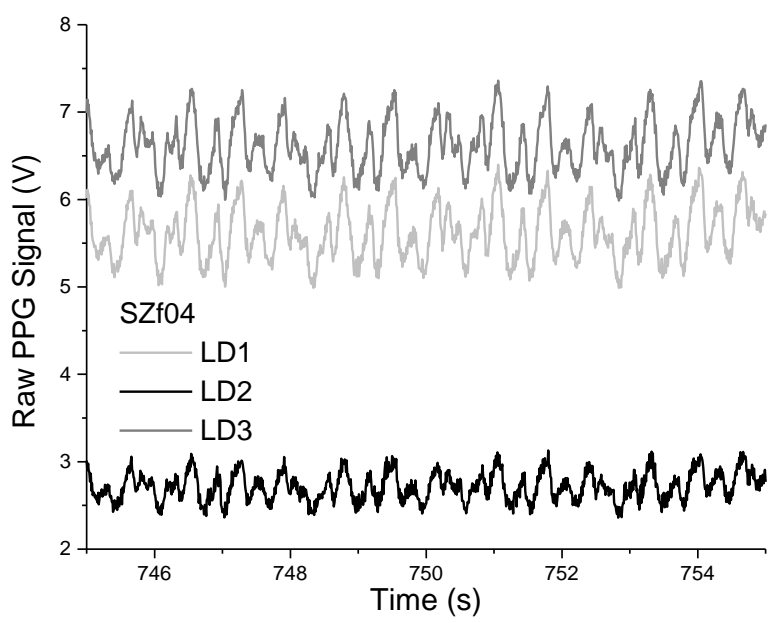

(d)

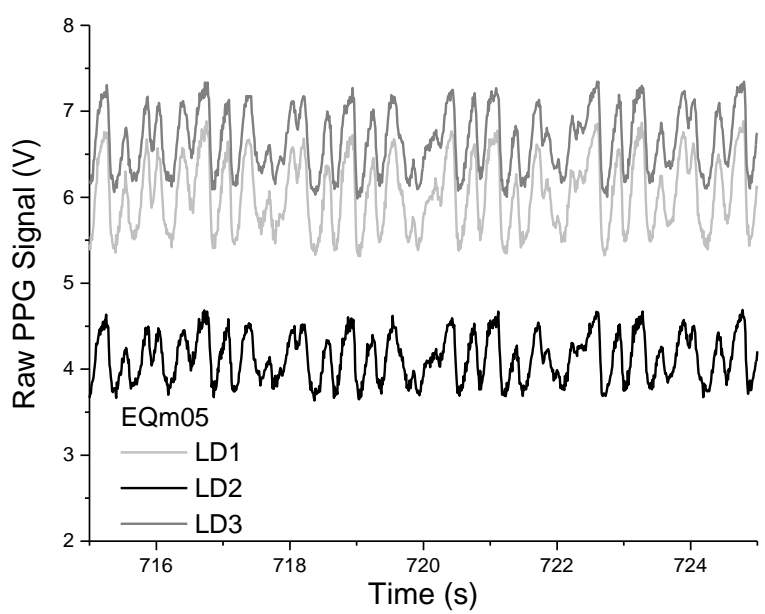

Figure 7: Raw transmittance photoplethysmographic signals recorded in 10-second time intervals using the transmittance optical sensor with the laser diodes LD1, LD2 and LD3 from the female (a, b, SZf04) and male (c, d, EQm05) athletes of figure 5.

\section{CONCLUSION}

The heart rates (beats per minute, bpm) obtained with our new three NIR laser diodes based sensory system over maximal exercise tests by treadmill ergometer performed in female and male athletes, agreed with those obtained by electrocardiography, despite movement artifacts. Further experiments with more athletes and analysis of its results with respect to the established techniques are in course. It will also let to refine the processing parameters and to demonstrate the improved performance of our photoplethysmographic multi-NIR-LD system, even in conditions of strong movements. 


\section{ACKNOWLEDGMENTS}

This work has been partially supported by the grants from the Spanish Ministry of Education and Science CSD 04/EPB 10/03 and CSD 01/EPB 10/04. Spanish Program "Ramón y Cajal" supports SMLS.

\section{REFERENCES}

1. A.A.R. Kamal, J.B. Harness, G. Irving and A.J. Mearns, "Skin photoplethysmography - a review", Comp. Methods Programs Biomed. 28, 257-269 (1989).

2. K. Nakajima, T. Tamura and H. Miike, "Monitoring of heart and respiratory rates by photoplethysmography using a digital filtering technique" Med. Eng. Phys. 18, 365-372 (1996).

3. L. G. Mengelkoch, D. Martín, J. Lawier, "Review of the principles of pulse oximetry and accuracy of pulse oximeter estimates during exercise", Physical Therapy 74, 40-49 (1994).

4. J. T. B. Moyle, "Uses and abuses of pulse oximetry", Arch. Dis. Child. 74, 77-80 (1996).

5. W. W. Lee, K. Mayberry, R. Crapo and R. L. Jensen, "The accuracy of pulse oximetry in the emergency department", Amer. J. Emerg. Med. 18, 427-431 (2000).

6. W. G. Zijlstra, A. Buursma and W. P. Meeuwsen van der Roest, "Absorption spectra of human fetal and adult oxyhemoglobin, deoxyhemoglobin, carboxyhemoglobin and methemoglobin”, Clin. Chem. 37, 1633-1638 (1991).

7. W. G. Zijlstra and B. Oeseburg, "Definition and notation of hemoglobin saturation", IEEE Trans. Biomed. Eng. 36, 872 (1989).

8. J. Achten, A. E. Jeukendrup, "Heart rate monitoring: applications and limitations", Sports Med. 33, 517-538 (2003).

9. H. B. Nielsen, "Arterial desaturation during exercise in man: implication for $\mathrm{O}_{2}$ uptake and work capacity", Scand. J. Med. Sci. Sports 13, 339-358 (2003).

10. R. Giannetti, J. P. Silveira Martín, M. L. Dotor, D. Golmayo, P. Martín, F. Miguel-Tobal, A. Bilbao, S.M. López Silva, "Oxygen saturation measurements in athletes attaining maximal exertion conditions", Proc. 21th IEEE Instrum. Meas. Tech. Conf. vol. 1, 740-744 (2004).

11. Y. Yamaya, H. J. Bogaard, P. D. Wagner, K. Niizeki, and S. R. Hopkins, "Validity of pulse oxymetry during maximal exercise in normoxia, hypoxia and hiperoxia," J. Appl. Physiol. 92, 162-168 (2002).

12. R. Giannetti, S. López, M. L. Dotor, J. R. Sendra, J. P. Silveira, and F. Briones, "An innovative signal processing algorithm for near infrared laser-based pulse oximeter," in Proceedings of 10th International Symposium on Development in Digital Measuring Instrumentation, Naples, Italy, September 17-18, 1998, Pasquale Daponte, Massimo D’Apuzzo, and Antonio Langella, Eds. IMEKO TC-4, Sept. 1998, pp. 153-156. 